\title{
Formación contínua en educación artística: sus efectos transformadores en la educación infantil y primaria
}

\section{Continuing education in artistic education : its transformative effects in early infant and primary education}

FORNÉ, E. ${ }^{1}$

\section{Resumen}

Dentro de la variedad de áreas de conocimiento que constituyen sus etapas de educación infantil y primaria, la educación artística constituye una opción muy efectiva para potenciar de manera integral todas las actividades sensibles, expresivas, creativas y reflexivas del alumnado, en este orden de ideas el presente articulo está orientado a exponer los resultados aportados del estudio de caso y mostrar los aportes conceptuales, pedagógicos y didácticos que producen este tipo de procesos proporcionados a docentes pertenecientes a los claustros de dos centros de educación infantil y primaria. Se muestra como con la formación continua para docentes basada en las artes es esencial para desarrollar actividades novedosas e innovadoras con el profesorado convirtiéndose en un instrumento mediante el cual se logra la transformación de la cultura de los propios centros.

Palabras clave: educación artística, educación infantil y primaria, formación continua, transformación de centro.

\begin{abstract}
Within the variety of knowledge areas that constitute the stages of nursery and primary education, Artistic Education is an effective option to comprehensively enhance all the sensitive, expressive, creative and thoughtful activities of students. In this order of ideas the article is aimed to set forth the results of the case study and to explain the conceptual, pedagogical and didactic contributions produced by these type of processes provided to nursery and primary education teachers belonging to two schools. It shows how continuous training based on Art Eeducation is essential to develop original and innovative activities for teachers and so becoming an instrument through which the transformation of the schools culture is achieved.
\end{abstract}

Key words: artistic education, nursery and primary education, continuous training, school transformation.

\section{Introducción}

Esta investigación analiza los beneficios que producen los procesos de formación continua en educación artística en la práctica de docentes de centros de educación infantil y primaria, a fin de ampliar su enfoque pedagógico y el conjunto de experiencias educativas que éstos ofrecen a su alumnado; en cuanto a que la educación artística

${ }^{1}$ Universitat Autònoma de Barcelona. UAB. Email: eforne@xtec.cat 
tiene un potencial transformador para el alumnado, sin embargo, en este estudio se focaliza en el potencial transformador que la formación continua en esta área proporciona en los docentes y en los centros educativos.

En este sentido, aunque actualmente se reconoce la importancia de la Educación Artística dentro de la Educación Infantil y Primaria, una de las principales dificultades que enfrenta el profesorado en el momento de comenzar su trabajo en esta área es el desconocimiento profundo de sus funciones, conceptos, procedimientos y utilidades en la formación integral del alumnado; en cuanto a ello, Acaso (2009) ha planteado la contradicción que experimentan los profesores que se inician en la docencia del arte: al enfrentarse a sus primeras clases y no conocer formas distintas de enseñar el arte, recurren a la reproducción de las metodologías con las que aprendieron y con las que muchas veces no están; ni han estado, de acuerdo. La autora plantea: “¿Cómo puede ser que la mayoría de los profesores enseñan de la misma forma con la que han sido enseñados aun cuando literalmente aborrezcan dicho sistema?" (Acaso, 2009, p.16).

Asimismo, esta investigación nace de una de las principales problemáticas que los docentes de Educación Infantil y Primaria enfrentan respecto a la Educación Artística: la falta de apropiación, conocimiento, exploración y reflexión de sus elementos, conceptos, modelos, funciones, procedimientos y finalidades, tanto en su aplicación con el alumnado como en coherencia con el resto del claustro. Se considera crucial la repercusión de este problema dado que como afirma Acaso, (2009) "una educación artística nueva necesita, por delante de todo lo demás, nuevos profesionales, conocedores de la materia, críticos, conscientes de su hacer político, en consonancia con los tiempos que corren" (p.123).

En este ámbito, para Aznárez (2018) actualmente existe una evidente necesidad de repensar y rediseñar la educación artística ya que ha podido corroborar que la gran mayoría de actividades de Educación Artística que los estudiantes desarrollan están ligadas a los trabajos manuales. Estas prácticas, según el autor, carecen de significatividad y relevancia desde el punto de vista cognitivo y técnico, son realizadas de manera uniforme por todos los estudiantes, y usualmente están centradas en las efemérides (día de la madre, Navidad...) o la realización de material de apoyo visual de otras asignaturas (mapas 3D, dibujos de plantas, esculturas para la asignatura de Literatura...). Estas actividades son acompañadas regularmente con ejercicios tradicionales basados en "Fichas/láminas de Internet o de libros de texto, para colorear/recortar" (Aznárez, 2018, p.18) y siguen promoviendo la repetición mecánica de acciones que muchas veces carecen de sentido y conexión para el niño con la realidad en que vive, a pesar de tener la apariencia moderna que la tecnología digital les aporta bajo un enfoque instructivo.

Aznárez (2018) enfatiza que en muchas ocasiones este tipo de actividades se llevan a cabo sin establecer metas y objetivos claros y, menos aún, vinculándolas con algún aspecto de la realidad del alumnado. Ello las hace tediosas, aburridas y poco motivadoras promoviendo así, paradójicamente, el alejamiento y la apatía del alumno respecto al lenguaje artístico. Invitando al estudiante a hacer lo que desee:

Haz lo que quieras" o "lo que sientas". Se incita al alumno a que dibuje o pinte lo que le venga en gana, para así desarrollar su creatividad. Sin un objetivo claro, como si eso bastara para desarrollar la creatividad y para enriquecer el "mundo interior". A veces sólo es una excusa para "relajar" y "dar un respiro" a los niños -o a sus docentes- entre las materias "serias. (Aznárez, 2018, p.18)

En este contexto, esta concepción erróneamente confiere un aura de frivolidad y poca importancia a la expresión artística en el medio educativo. Según Aguirre (2005), "todavía pesa mucho la inercia de las manualidades, la libre expresión y la vinculación de la formación artística a los supuestos "dones naturales " de los estudiantes hacia el arte" (p.52). En relación a ello, el mismo autor comenta que "la definición del objeto propio de estudio de la educación artística y su ubicación en el mapa de las disciplinas escolares” (p.54), constituiría uno de los más 
relevantes. Esto ha llevado al alumnado a perder el interés por su experimentación o incluso a desvalorizar sus resultados debido, fundamentalmente, a dos motivos: El primero, expuesto anteriormente, radica en que se trata de actividades sin planificación y objetivos claros, por lo que están descontextualizadas de su vida real y carecen de valor. El segundo de los motivos es producido por la tendencia a clasificar la producción artística de la escuela como meras manualidades sin objetivos y ejercicios aficionados sin interés, lejanos de la categoría de genialidad artística.

Dado lo antes expuesto, a partir de los comentarios realizados por decenas de profesores y profesoras de Educación Infantil y Primaria que acuden a los cursos de formación continua, constatamos que todas estas circunstancias son excesivamente frecuentes en los centros escolares. Este hecho dificulta que el docente de Educación Infantil y Primaria pueda generar verdaderos procesos de aprendizaje significativos a través de la Educación Artística, ya que debe conocer todas sus capacidades desde el acercamiento profundo a ésta en el que se debe propiciar, paralelamente, la experimentación sensible, la introspección emocional y la reflexión crítica.

Es aquí donde resulta crucial el estudio de la relación entre la Educación Artística, la formación continua para docentes y la observación de los procesos que se dan en escenarios formativos, como los programas del CESIRE y sus itinerarios artisticos, diseñados con el fin de dotarlos de herramientas conceptuales, metodológicas y procedimentales y, a su vez, involucrarlos en sólidas reflexiones sobre los alcances y finalidades de la Educación Artística en el contexto actual. Se considera muy relevante conceder atención a aquellos proyectos dirigidos a fomentar los procesos de "la enseñanza y el aprendizaje de las artes visuales y plásticas en los futuros educadores de la enseñanza primaria" e infantil, dado "que son ellas y ellos quienes primero deben familiarizarse con las nuevas tendencias para poder guiar a su alumnado" (Llonch Molina, Martín Piñol \& Santacana Mestre, 2017, p.319).

Sin embargo, aunque la Educación Artística ofrece una gran facilidad para transversalizar los procesos de aprendizaje y para adaptarse a los diferentes contenidos académicos, requiere tanto de un proceso de conocimiento del ámbito como de una adaptación responsable proporcionando al docente las competencias para que logren en su ejercicio pedagógico la inserción en las actividades curriculares propuestas en el sistema educativo catalán, planteándose en este proyecto la posibilidad de generar procesos de cambio metodológico en los centros analizados. Desde lo que se considera como el segundo escenario de análisis de este estudio, denominada educación continua.

En este sentido, se percibe el proceso de educación continua como parte del proceso formativo dirigido al profesorado que, en calidad del ejercicio de sus funciones, se debe reconocer la eterna necesidad de actualizarse, permitiéndose espacios de capacitación y aprendizaje en los que pueda incorporar a su ejercicio las diferentes novedades que aparecen en materia de aprendizaje. Se entiende que el presente estudio es relevante en la actualidad y que responde a unas necesidades expresadas por parte de la comunidad docente, ya que se fundamenta en la importancia que tiene el proceso de educación continua dirigido específicamente al profesorado. El proceso de educación continua del profesorado presenta una oportunidad infranqueable para la manifestación de expresiones artísticas que dinamicen su proceso de aprendizaje y generen procesos reflexivos, que no únicamente promuevan la expresión creativa en sus actividades cotidianas, sino que también promuevan el cambio metodológico en los centros. Esto implica un proceso de transformación desde las artes, que remite a integrar "la coherencia con la necesidad de superar modelos transmisivos hacia modelos transformativos" (Kennedy, 2005, p.45) y co-constructivos" (Monereo, 2010, p.87). Dentro de los elementos que preceden esta acción, se encuentran las estrategias pedagógicas que el docente lleva a cabo para tener la certeza que las actividades que realice tengan un propósito y poder hacer prosecución de ello.

Cabe destacar que si bien "hoy en día existe un interés mayoritariamente generalizado de encaminar la enseñanza hacia la creatividad" que sirva para "formar a los alumnos mediante preceptos significativos que les 
permitan reaccionar y solucionar conflictos y situaciones" (Vilaboa, 2005, p.1), paradójicamente la reducida formación de algunos docentes del área de Educación Artística "ha sido muy pobre y no ha contribuido a mejorar su auto concepto en lo tocante a la creatividad o las capacidades artísticas" (Aznárez, 2018, p.18). Se torna indispensable potenciar la formación del personal formador para así conseguir que a través de nuevas estrategias metodológicas se contribuya a una educación de calidad (Criteris, instruccions i orientacions. Formació permanent del professorat. Departament d'Educació, 2019, p.8).

Sintéticamente, esta investigación busca abordar la necesidad identificada en el claustro, que necesita contar con medios de apoyo en su objetivo de promover paralelamente la cultura, las expresiones artísticas y la innovación de forma coordinada y coherente con la identidad del centro. Por otra parte, pretende también abordar la necesidad de los docentes por adquirir herramientas conceptuales, metodológicas y sensibles que le ayuden a potenciar tanto su capacitación artística como la de sus alumnos y alumnas, quienes, en definitiva, son sus principales destinatarios. Finalmente, este estudio se propone aportar a los propios estudiantes, quienes requieren encontrar en la experiencia educativa, actividades orientadas al desarrollo de sus capacidades sensibles, reflexivas y, sobre todo, creativas.

\subsection{Objetivos}

Analizar cómo repercuten en la práctica pedagógica de los docentes de educación infantil y primaria los procesos de formación continua en el contexto de transformación de centro a través de la educación artística.

Los objetivos específicos, definidos a partir del objetivo general, se concretan en:

Indagar los referentes teóricos que sustentan los procesos académicos relacionados con la dinamización de los procesos de educación continua a través de la educación artística.

Mostrar los resultados de experiencias académicas fundamentadas en el uso de la educación artística en los procesos de formación dirigidos a docentes.

Contrastar la influencia que tiene el desarrollo de procesos de educación continua, centrados en la educación artística para docentes, en el desarrollo de los procesos formativos de sus estudiantes de educación infantil y primaria en el contexto de transformación de centro a través de las artes.

\section{Metodología}

El presente estudio se inscribe en la perspectiva cualitativa interpretativa de investigación tal y como se explicita a continuación. Para Husserl (1859-1938), el paradigma interpretativo es un camino para aproximarse a la realidad donde "la verdad no reside en el juicio sino en sí misma, la evidencia es el percatarse de la coincidencia entre la significación y el significado, de la perfecta correspondencia entre la afirmación y el hecho" (1966, p.3).

Esta posición implica que el investigador comprende la realidad a través de un proceso de conocimiento e interpretación, relacionando el mundo real con el mundo vital. Tal como señala el autor, esto permite captar "la esencia del fenómeno (...) analizando el significado que el sujeto le da a sus acciones" (Husserl, 1966, p.33). Por tanto, el paradigma interpretativo se fundamenta en las características del objeto de estudio y de los enunciados interrogativos que se realizan sobre él. Se trata de un proceso cuyo propósito fundamental es el de generar teoría a través de una estrategia en espiral, una lógica de escenarios de análisis que permitan comprender el objeto de estudio.

Su elección para este estudio se fundamenta entonces en que se orienta hacia "la construcción de conocimiento acerca de la realidad social y cultural a partir de la descripción e interpretación de las perspectivas de los sujetos involucrados" (Rojas, 2014, p.57), facilitando el estudio de los procesos artísticos de forma que sean descritos 
explícitamente y dando paso a su comprensión global. A partir de estas consideraciones, y como fase preliminar a la investigación, se plantea la posibilidad de concretar este estudio teniendo en cuenta las siguientes características: (1) El foco de la investigación tiene carácter exploratorio y descriptivo; (2) El diseño es emergente, se elabora sobre la información recogida; (3) La recogida de datos tiene lugar en situaciones naturales, no controladas; (4) Enfatiza el papel del investigador como instrumento principal de la investigación; (5) Los métodos de recogida de la información son cualitativos, es decir, de naturaleza interactiva; (6) El análisis de datos es inductivo: categorías y patrones emergentes se construyen sobre la base de la información obtenida; (7) El informe de investigación sigue el modelo del estudio de casos (Maykut \& Morehouse, citado por Latorre et al., 2005, p. 200).

El estudio de caso permite la exploración a profundidad del fenómeno estudiado en sus contextos naturales (Bodgan \& Biklen, 1982; Vázquez \& Angulo, 2003), comprendiendo la forma en que los participantes significan la situación objeto de estudio (Sandín, 2003). El estudio de caso parte de la descripción de los sujetos para aportar el mejoramiento de las prácticas educativas (Ballester, Nadal \& Amer, 2014; González, Arquero \& Hassal, 2014) relacionadas con las experiencias de educación continua al profesorado, fundamentadas en las experiencias artísticas.

En esta investigación, concretamente se analiza la relación entre educación artística y formación continua por medio de un estudio de caso aplicado desde la observación de los escenarios donde se producen procesos formativos desarrollados por el CESIRE de la Generalitat de Catalunya, Departament d'Educació (2020).

Por otra parte, también se asume que el diseño es emergente y concebido en forma de cascada: "un tipo de investigación cuyos resultados no provienen de instrumentos cuantitativos ni son analizados mediante tratamiento estadístico" (Rojas, 2014, p.87). En este paradigma de investigación los resultados parten de la comprensión y de un proceso de reflexión de los resultados. A partir de esta metodología cualitativa se buscará explorar los fenómenos en profundidad, analizar la información recabada e interpretar las manifestaciones de los sujetos a través de la interacción con ellos.

Gracias a esta metodología cualitativa sustentada en el estudio de caso se dará cuenta del desarrollo que se ha tenido en cada una de las sesiones en las que se implementará la educación artística en el desarrollo de los procesos de formación continua del cuerpo docente de educación primaria de los dos centros educativos seleccionados para el estudio: Escuela Joan Miró de Canovelles y la Escuela Ferrer i Guàrdia de Granollers.

\subsection{Instrumentos, técnicas y proceso para la recogida y análisis de la información}

Rodríguez \& Bonilla (2005) señalan que los datos en una investigación deben obtenerse por medio de instrumentos que recojan la información tal como la expresan los informantes clave. Además, es "fundamental registrarla de tal modo que puedan recuperarse de manera fácil y ordenada para su revisión y análisis" (p.158). La selección de las técnicas y el diseño de los instrumentos de recolección de información son determinantes, ya que han de complementarse entre sí y apuntar al desarrollo de los objetivos de la investigación.

Por tanto, para dar mayor relevancia a este proceso de recolección de datos y respondiendo a esa complementariedad se insta a integrar herramientas como la entrevista, como una técnica de intervención de apoyo para complementar la recolección de datos, basados en una de sus principales funciones, darle prioridad a la voz de los participantes, de esta forma se logra captar las necesidades de los centros a través de sus actores. A partir de las consideraciones precedentes, para este estudio se decidió implementar los siguientes instrumentos: entrevista, grupo focal y observación.

En este ámbito, los informantes elegidos para este estudio estuvieron conformados por: 
Escuela Ferrer Guardia. ART y Transformación a partir de un proyecto de centro. Granollers. SE. Vallès Oriental IMaresme, Curso 2017-18 Equipo piloto: 4 personas con el apoyo de la directora haciendo seguimiento desde el centro y puntualmente en la formación. Asimismo, participaron de la formación en el CESIRE durante el curso 2017-2018. Durante el curso 2018-2019 asistieron a la formación como alumnas, pero también como formadoras 18-19.

El mismo curso también participaron como formadoras y coordinadoras de la formación territorial del Vallès Oriental ofreciendo la Escuela como sede de la formación de algunas sesiones. Por lo que respecta a la formación en centro y transferencia a todo el claustro se llevó a cabo con todo el equipo piloto + directora curso transferencia en el centro: todo el claustro. En este sentido, también participó de la formación como asistentes y también como formadores de la Escuela Joan Miró. Arte y Tecnología de Canovelles SE. Vallés Oriental IMaresme.

Durante el curso 2018-2019 todo el claustro participó (23 docentes) de la formación en el CESIRE. Hicieron todas las cápsulas / 25 cápsulas/ $2 \mathrm{~h}$ - Todos acreditaron formación de 60 h. En la formación territorial también ofrecieron su centro para acoger algunas sesiones de formación. El equipo directivo y las especialistas de música y plástica también llevaron a cabo algunas sesiones como personas formadoras. En este contexto los informantes de las entrevistas fueron los siguientes:

Cuadro 1

Informantes de las entrevistas

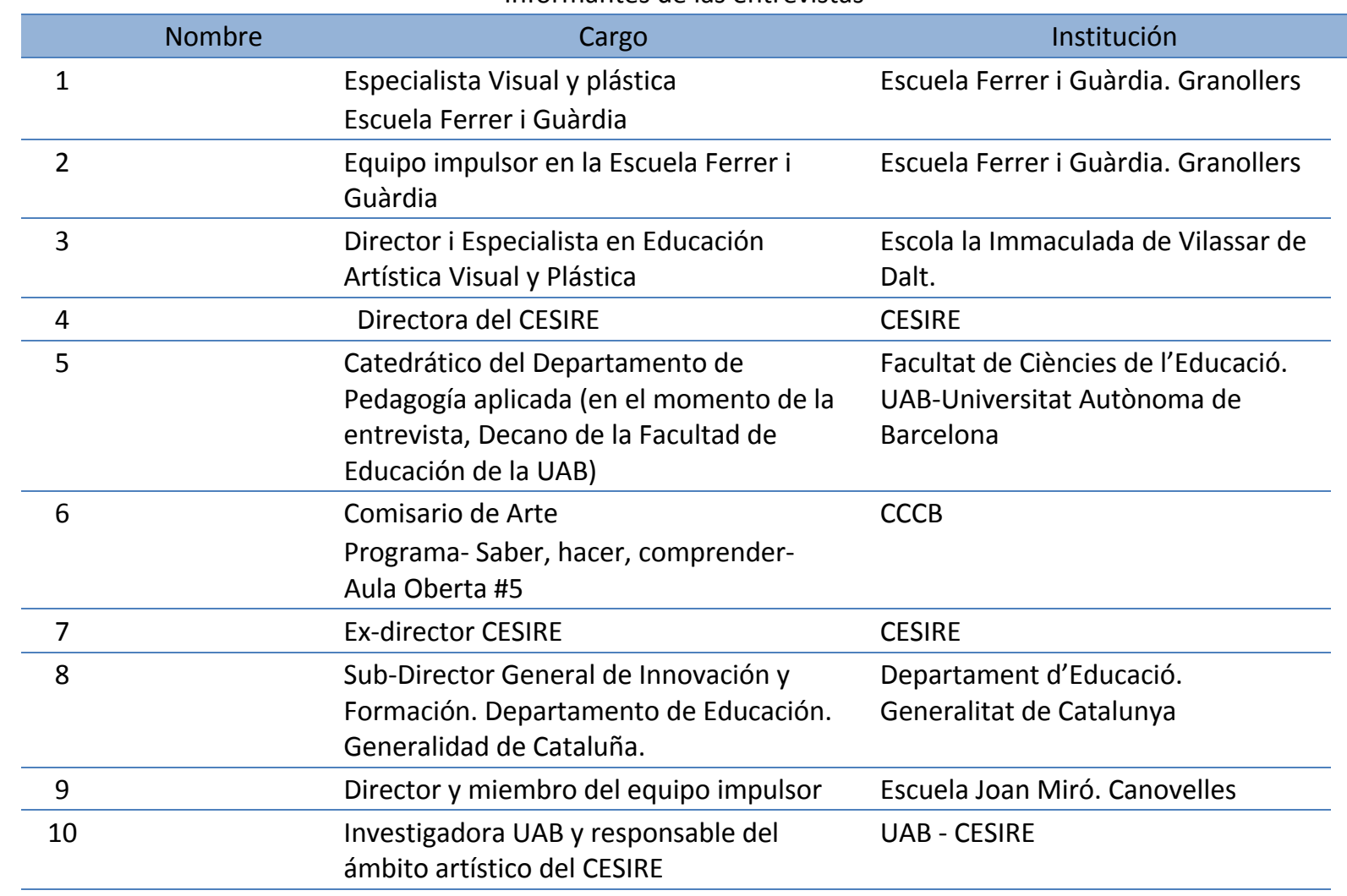

Fuente. Elaboración propia (2020)

Posteriormente se procedió al análisis de datos recopilados en el escenario de los programas de formación continua ofertados por el CESIRE para los dos centros educativos seleccionados para este estudio. Después de identificar las necesidades de los centros se procede a desarrollar las actividades de formación a través del Centro de Recursos Pedagógicos Específicos de Apoyo a la Innovación y la Investigación Educativa de la Generalitat de Catalunya. Departament d’Educació (2020) (CESIRE). Este es un centro específico de apoyo a la innovación y la 
investigación educativa y su finalidad es conocer la investigación en didáctica y educación, para promover y difundir sus resultados y adecuarlos a las necesidades del profesorado que los deberá transferir a la práctica docente. También tiene la función de diseñar y difundir actividades y recursos que ayuden al profesorado en su tarea innovadora, para mejorar los resultados escolares del alumnado.

Esta formación promueve el empoderamiento de los docentes y/o centros invitándolos a crear su Itinerario formativo a partir de la selección de las cápsulas o sesiones que más se adaptan a sus necesidades, intereses, habilidades o capacidades. De esta manera se les invita a configurar su propio currículum rizomático (Deleuze y Guattari, 2002), poniéndolo en relación al de los compañeros o centros y estableciendo relaciones flexibles, de colaboración y fomentando las estructuras relacionales horizontales, la creatividad, y la no centralidad en la toma de decisiones curriculares. Las cápsulas que se ofrecieron se muestran en la siguiente figura:

Figura 1

Estructura de los itinerarios artísticos
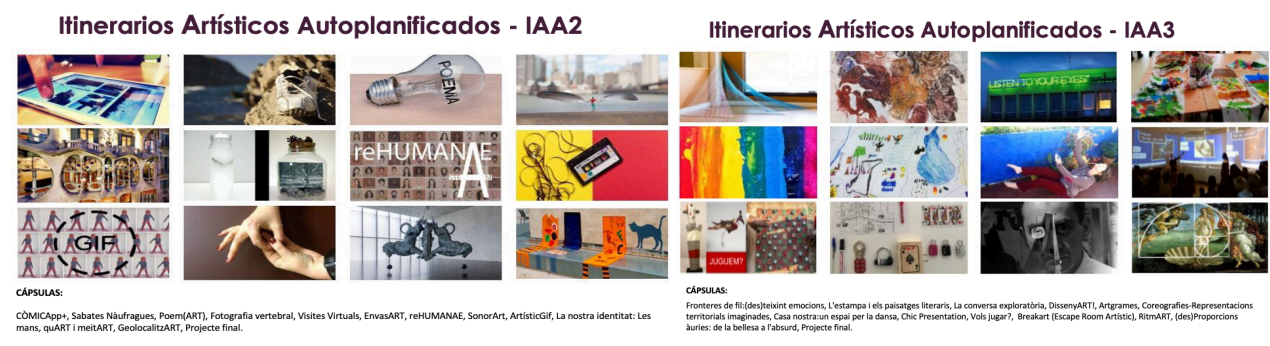

Itinerarios Artísticos Autoplanificados - IAA4
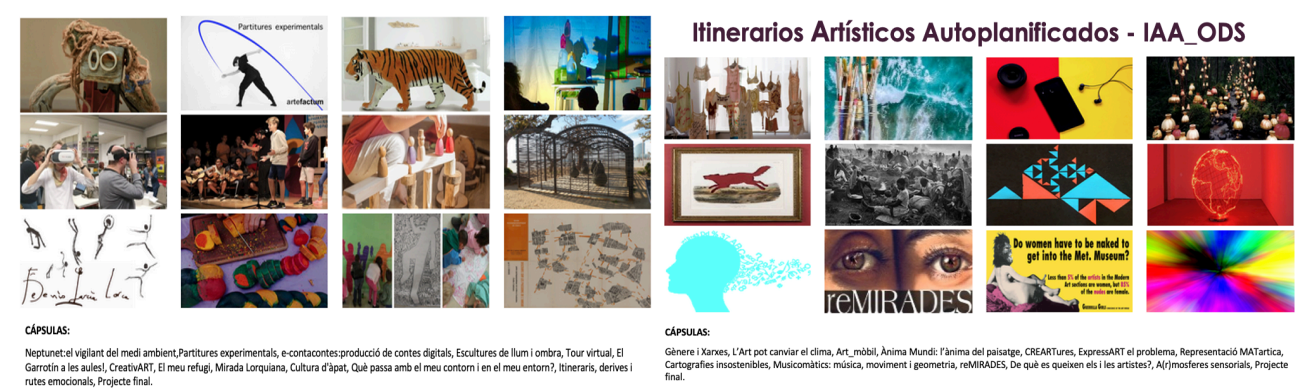

Fuente: elaboración propia. Detalle de las cápsulas que conforman cada uno de los 4 cursos que se ofrecen. (curso 2020-2021)

De acuerdo con las funciones de la institución ha impulsado varios proyectos en el ámbito de la educación artística: Ens transformem amb Art, Centres d'Art en Acció, Transforamció de centre a través de les Arts, Proyectos STEAM-ODS, etc, de los cuales remarcamos el proyecto de formación continua: Itineraris Artístics Autoplanificats y que se analizan en esta. Dicho análisis sirvió primeramente para enmarcar las líneas de acciones de los centros donde se han pilotado las acciones formativas y de transformación y que centrarán el estudio de caso de esta investigación. Por otra parte, sirvió para proveer de cuerpo teórico y de investigación educativa a los propios proyectos, y finalmente, este estudio servirá también para aportar datos y pautas para su réplica en otras experiencias similares tras evaluar su validez. El objetivo final de los dos proyectos es su transferencia a toda Cataluña. Para esto utiliza una metodología lúdico-participativa, que se desarrolla a través de los Itinerarios Artísticos Autoplanificats descritos por Generalitat de Catalunya, Departament d'Educació (2020) de este modo, cada uno de los itinerarios resultantes será diferente y más personalizado a los intereses de cada uno en función de las sesiones o cápsulas que haya escogido. 
Con base en estas consideraciones, las categorías de esta investigación se presentaron como escenarios de análisis a través de matrices, gráficos y análisis, que permitieron comprender las respuestas de los participantes. A partir de las entrevistas realizadas emergieron subcategorías a través de las cuales se desarrollaron las incidencias e interpretación de la información, para finalmente triangular la información y presentar los resultados. Este proceso realiza un muestreo teórico en donde el analista "colecciona, codifica, analiza los datos y decide qué datos coleccionar más adelante y en donde encontrarlos para desarrollar una teoría mejor a medida que la va perfeccionando" (Glaser \& Strauss, 2012, p.25). El mismo se desarrolló partiendo de la teoría que emerge a partir de los escenarios de análisis y culminó con la saturación teórica de los datos partiendo de las incidencias de los participantes.

Las categorías surgen a partir de la realización de una entrevista inicial, cuyos resultados se agruparon tomándose en cuenta los elementos comunes y no comunes, a partir de lo cual surgen los escenarios de análisis y subcategorías que se presentarán en el capítulo siguiente. Toda esta información se presenta a través de matrices de análisis en las que se registran los tres escenarios de análisis: procesos de formación continua en docentes, educación artística y experiencias académicas del claustro con sus respectivas subcategorías e incidencias.

Figura 2

Subcategorias e incidencias del escenario de análisis
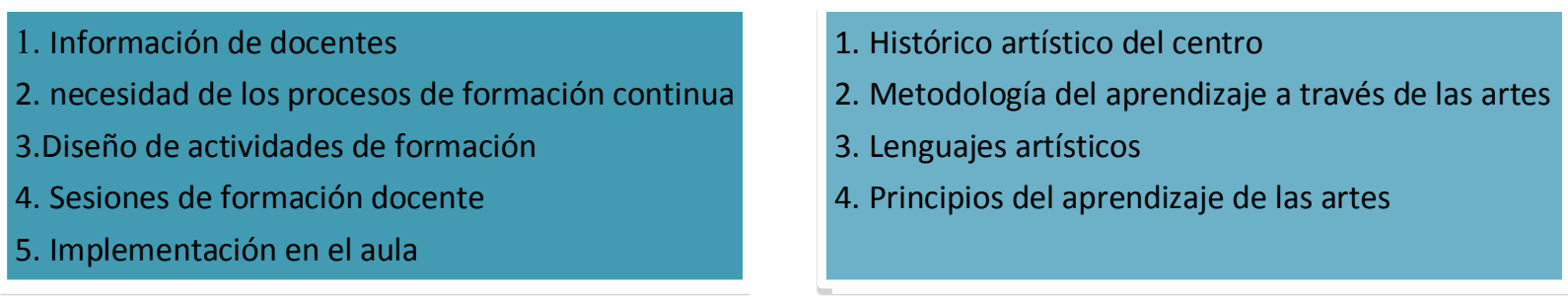
1. Cambios en el claustro
2. Redes educativas
3. Compromiso del claustro
4. Transformación e innovación educativa
5. Liderazgo del centro

Fuente. Elaboración propia (2020)

En este ámbito, para el análisis e interpretación de la información se escogieron distintos escenarios de análisis relacionados con los tres objetivos principales planteados en la investigación. En cada uno de estos escenarios, se discriminaron una serie de subcategorías, ilustradas por incidencias aportadas por los distintos participantes con la finalidad de manejar de manera ordenada la información. A continuación se presentan los escenarios de análisis y las categoría que emergieron de la información recabada de los informantes: 
Tabla 1

Escenarios de análisis y categorías

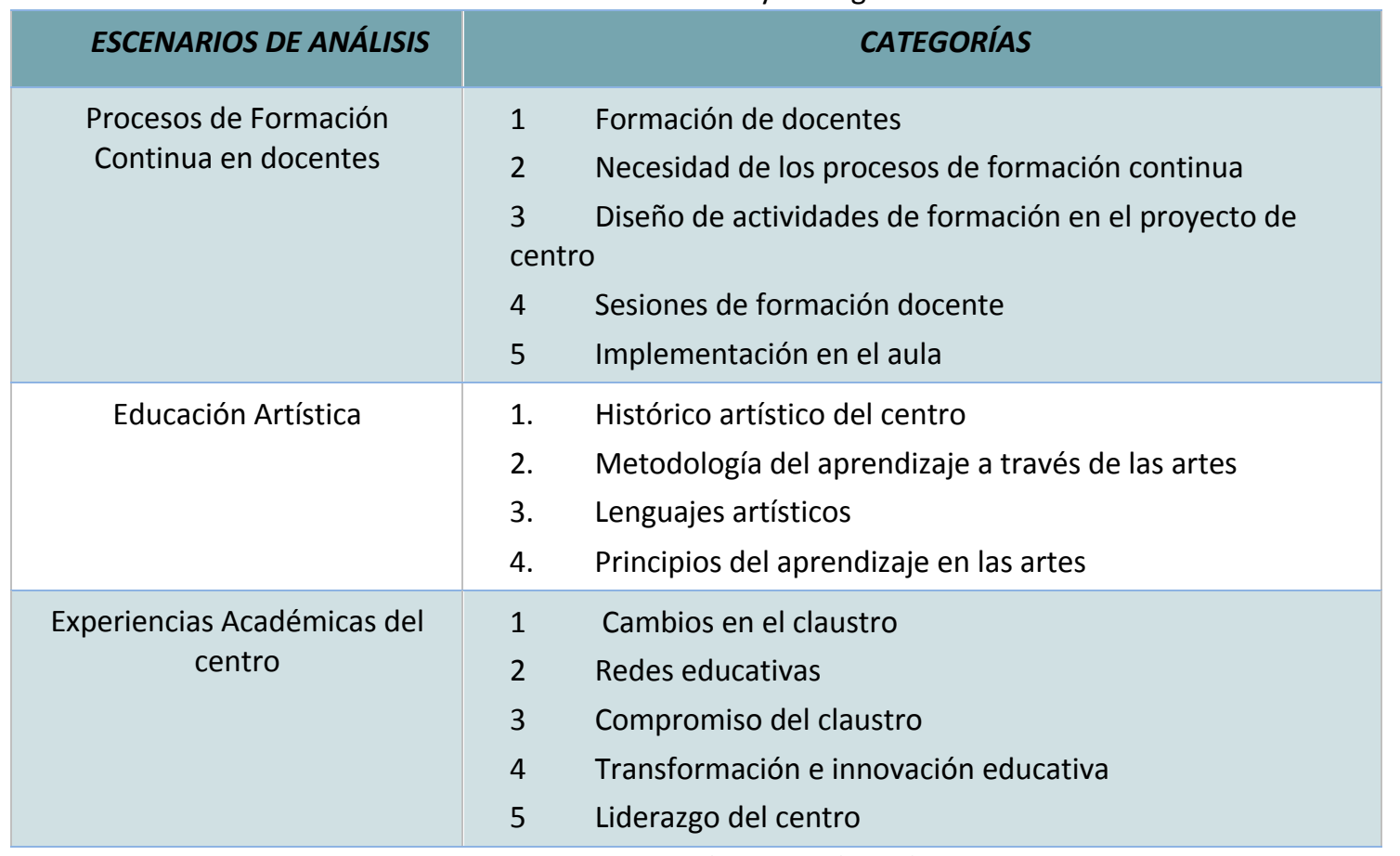

Fuente. Elaboración propia (2020)

Seguidamente se presentan los resultados y discusión a partir del trabajo obtenidos de las entrevistas, grupos focales y observaciones realizadas durante toda la investigación.

\section{Resultados y discusión}

En este apartado se presenta el análisis e interpretación de los resultados obtenidos a partir de las entrevistas realizadas a los participantes en la investigación. Con respecto al análisis de datos en una investigación cualitativa, Rojas (2014) señala:

El análisis cualitativo no debe verse como una fase final que ocurre una vez obtenido los datos, sino como un proceso continuo que comienza cuando el investigador se plantea las primeras interrogantes...Por esta razón es difícil explicitarla en pasos rígidos previamente establecidos. (p.145)

Es decir, pueden existir distintas metodologías de análisis e interpretación de resultados, sin embargo, dentro de una investigación cualitativa es el autor quien adecúa los métodos de acuerdo con la finalidad de su estudio. Rojas (2014) considera realizar una triangulación en la investigación para obtener resultados válidos y fiables del análisis de contenido: "la triangulación de fuentes permite contrastar la información obtenida de diferentes sujetos (...) acerca de un tema” (p.166). En la presente investigación, luego de haber presentado los escenarios de análisis con sus respectivas subcategorizaciones se realizó una triangulación de diferentes fuentes: lo que contempla la teoría, lo que expresaron cada uno de los participantes y la interpretación de la autora de esta investigación. 
Para presentar los resultados e interpretación, se realizó previamente el procesamiento de la información obtenida a través de una serie de tablas elaboradas a partir de las entrevistas realizadas por la autora, las expresiones son tomadas de los participantes tal cual como ellos las manifestaron. A continuación, se presenta una síntesis gráfica de los resultados:

Figura 3

Procesos de formación continua en docentes

Procesos de formación continua en docentes

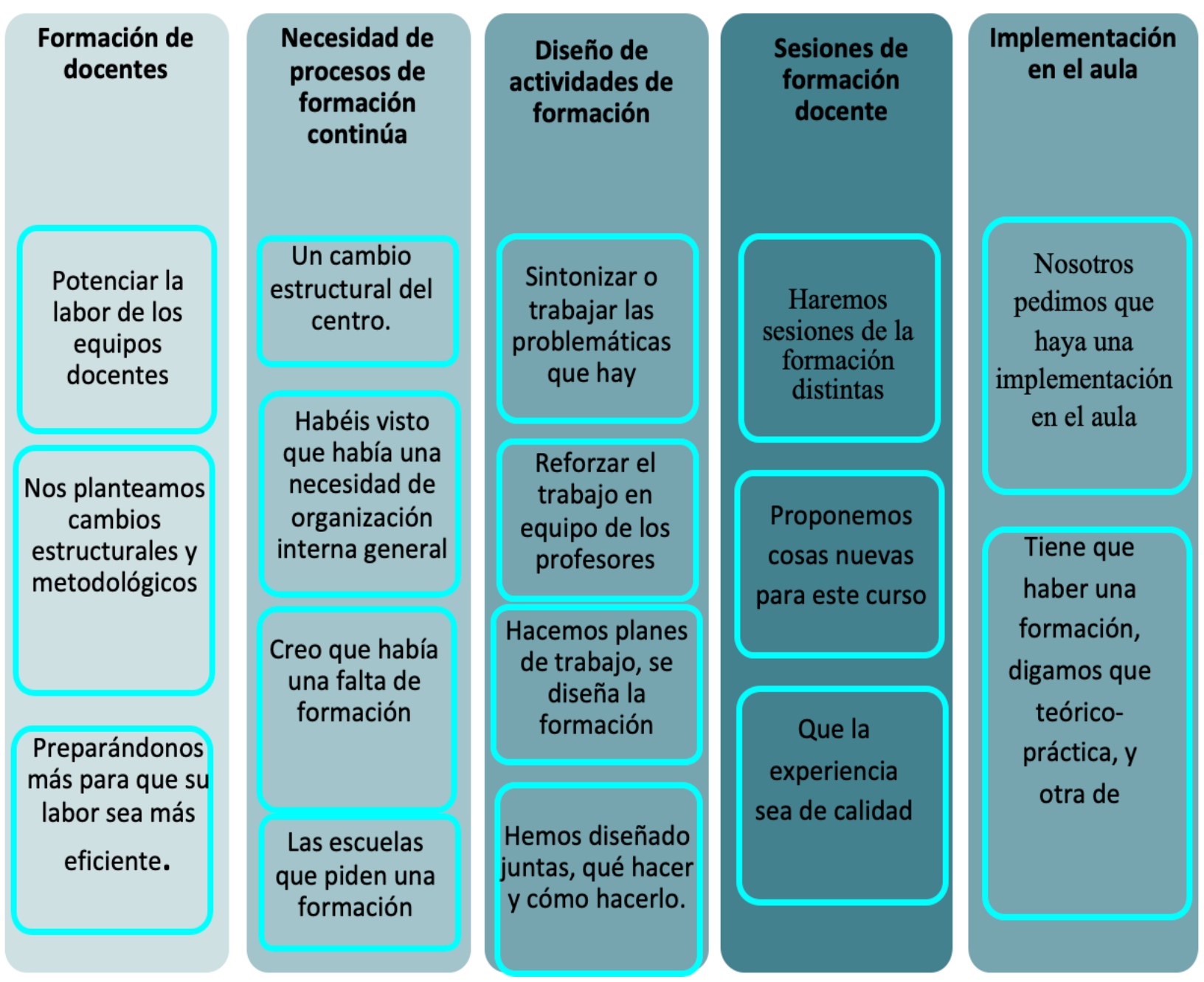

Fuente. Elaboración propia (2020)

Relacionando esta reflexión con la opinión de los participantes, se puede interpretar que estos entienden que el arte es el motor para producir el conocimiento y a través de los itinerarios, era posible profundizar en su conocimiento y experimentación. 
Figura 4

Educación artística

\section{Educación artística}
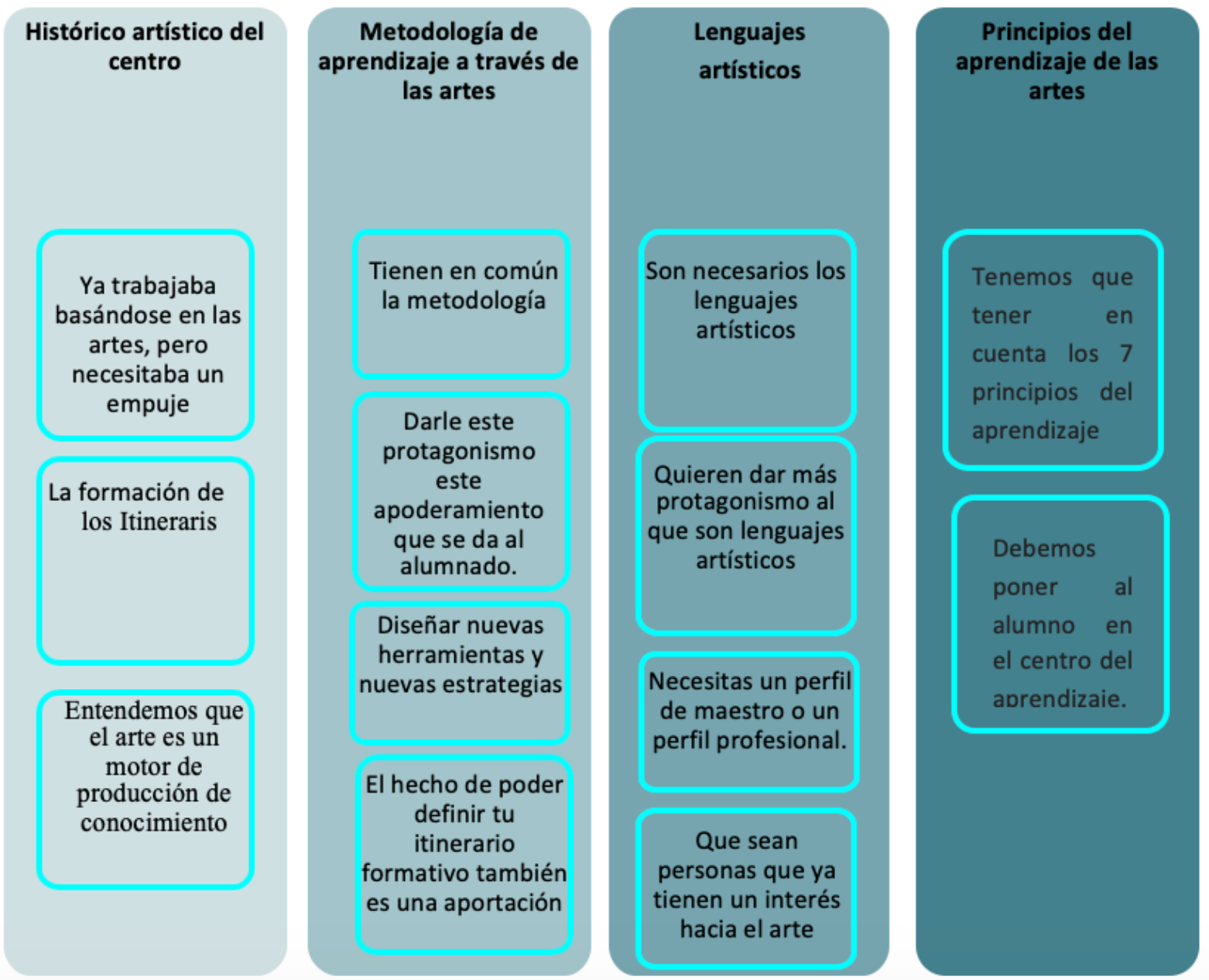

Fuente. Elaboración propia (2020)

En este escenario de análisis se destaca prioritariamente la necesidad e importancia de fortalecer la actividad docente a través de las experiencias académicas y las artes. 
Figura 5

Experiencias académicas del centro

\section{Experiencias Académicas del Centro}
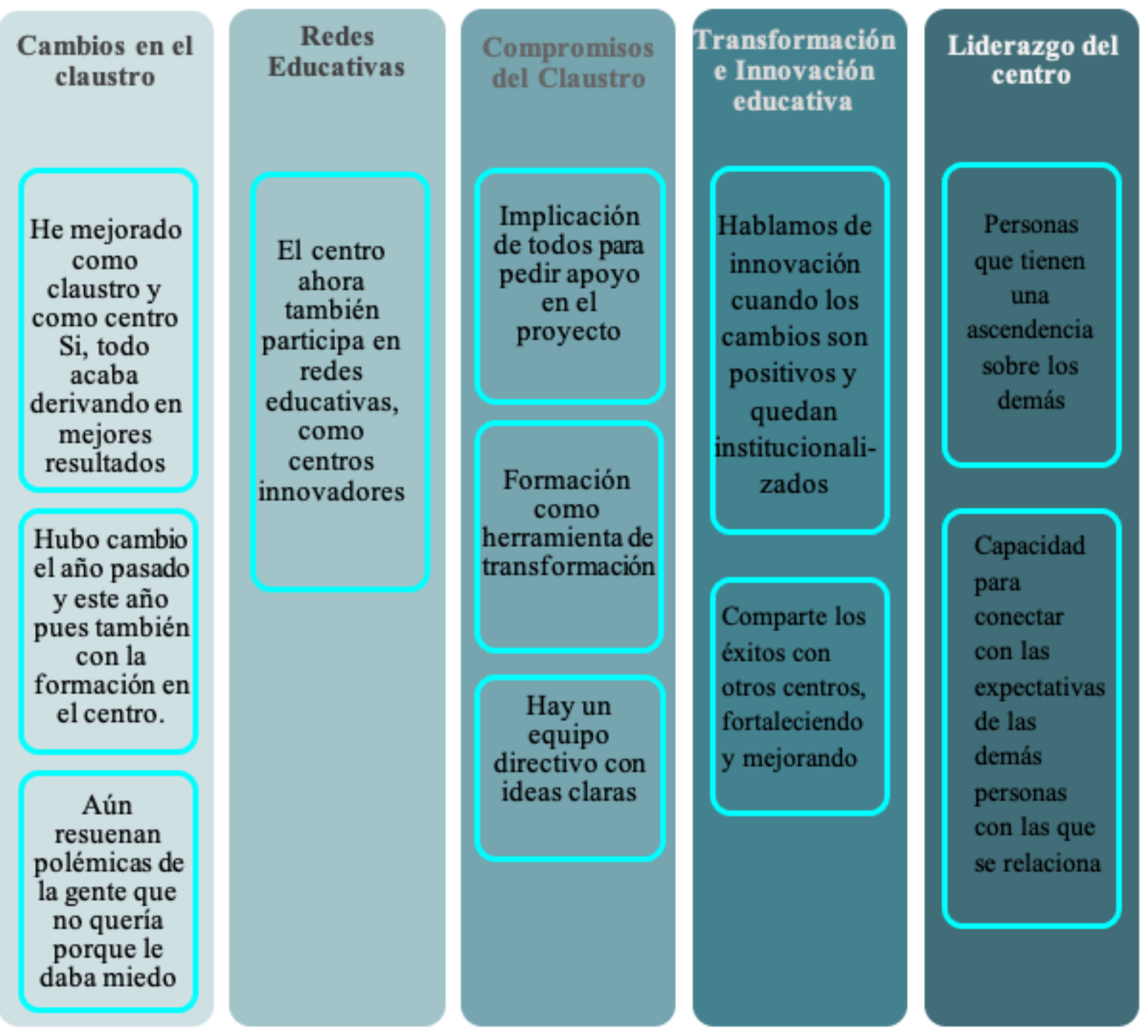

Fuente. Elaboración propia (2020)

\subsection{Discusión}

A partir de la respuesta de los participantes se evidencia que la formación continua de los docentes es necesaria con la finalidad de instaurar cambios estructurales y metodológicos en los centros educativos. En este ámbito, algunos participantes consideraron que faltaba formación para ajustarse a nuevas realidades y elaborar planes de trabajo pertinentes: consideran que se debe integrar la teoría y la práctica para que la educación sea de calidad y sea aplicable en las aulas. En este ámbito, la formación ha tenido muy buena acogida por parte del profesorado y los centros educativos. Esto se debe, por un lado, a la flexibilidad y, por el otro, es una respuesta a una demanda de un grupo de profesorado que tenía necesidad de una formación de este estilo, correspondiendo al profesorado su materialización en la propuesta curricular y en las programaciones de aula. 
Dicha materialización, se observa con la planificación de las sesiones de formación docente con el apoyo de todo el equipo del centro para la inserción en las actividades curriculares propuestas en el sistema educativo español, planteándose en este proyecto la posibilidad de generar procesos de cambio a través de las artes; facilitando el espacio para que el docente tome conciencia de su condición de eterno aprendiz y se adentre en la comprensión de sus fortalezas y debilidades. El profesor de hoy se enfrenta a ejercer como "mediador cultural y proporciona a los alumnos perspectivas de evolución y criterio en el medio social" (Bamford, 2009, p.8)- Aplicado a la educación primaria, esto permite que los niños y niñas internalicen y asuman su cultura en su entorno social. Asimismo, se proponen las posibles estrategias a utilizar en los procesos de formación continua docente: el diario de campo, el debate reflexivo, la redacción de casos, indagación en el aula, registros escritos, documentos personales, "portafolios", metáforas, mapas conceptuales, reflexión sobre la acción y comunidades virtuales" (Vaillant \& Marcelo, 2015). Todas estas técnicas expanden un panorama amplio que implica, a través de sus diferentes posibilidades, múltiples formas de actuación que permiten darle una transformación a los procesos de educación continua.

En el segundo escenario de análisis, denominado "Educación Artística", se profundiza en relación con el histórico artístico del centro en cuanto a sus experiencias en el campo de las artes, metodologías aplicadas, lenguajes artísticos y principios de aprendizajes de las artes. A través de esta entrevista, los participantes enunciaron aspectos teóricos relacionados que impactan directamente en la formación docente. Concretamente se refirieron al uso de la educación artística en el desarrollo de los procesos de educación continua para docentes, aportando a través de la educación artística los elementos necesarios para dinamizar la formación del profesorado de forma que pueda adentrarse en su proceso de redescubrir pedagógico y explorar sus habilidades. Señalaron, además, que se hace imprescindible en el desarrollo de procesos educativos artísticos que aporten a la sensibilización de la población frente a los elementos conceptuales, lograr que descubran este mundo de forma creativa.

Todo ello con la finalidad de superar aquellos modelos tradicionales conductista de estímulo respuesta e ir a un modelo que con el apoyo de las artes desarrolle de forma creativa en los niños y niñas las habilidades y destrezas en este campo; esto es "superar modelos transmisivos hacia modelos transformativos" (Kennedy, 2005, p.45). Es importante destacar la consideración de Rivera (2013), quien hace referencia al potencial de la educación artística para despertar los sentidos del ser humano, convirtiéndolos en seres más sensibles y ayudándolo a comprender la forma de pensar y actuar de otros. Esto aporta al mejoramiento de la estructura social, definiéndose como "un campo de conocimiento que busca potenciar y desarrollar la sensibilidad, el pensamiento creativo, la expresión simbólica en los contextos interculturales, representados en múltiples lenguajes: visual, corporal y auditivo" (Rivera, 2013, p.321).

En el tercer escenario de análisis, correspondiente a las Experiencias Académicas del centro, los participantes afirman que con la formación continua de docente a través de las artes han mejorado como centro, expresando el temor que manifestaron los mismos en relación a los cambios que se avecinaban.

Esta idea se encuentra en concordancia con Eisner (2002), quien plantea que la educación artística provee al centro un lenguaje que da valor la experiencia basada en una nueva forma de pensar la subjetividad, tanto individual como colectiva, y que utiliza estrategias narrativas que permiten reforzar las formas de relato clásicas complementándose gracias a las posibilidades que ofrecen también las tecnologías digitales. En este contexto, Eisner (2007), quien estudia a las escuelas y a las instituciones educativas de forma crítica, resalta su capacidad de permanecer invariables en el tiempo, de negarse a la posibilidad de transformarse, aportando en su negación a la creación de múltiples formas de analfabetismo. Para proponer desde su perspectiva, fundamentada en el pensamiento complejo, que las escuelas sean vistas como culturas y que como tales se permita la capacidad de 
transformarse en la interacción con los sujetos, con sus necesidades, su capacidad de crecer, cambiar (Eisner, 2007, p.27).

Por ello, uno de los elementos innovadores es que a través de las redes educativas los centros pudieron intercambiar experiencias en el campo de las artes y la formación, que fueron innovadores en los procesos de la calidad de la enseñanza. Eisner (2007) señala también que "Si los maestros y los directivos escolares no entienden el cambio o no llegan a comprometerse con él, no cambiarán" (p.27). Por ende, el proceso de actualización al profesorado a través de la educación continua es un proceso que se encuentra legalmente establecido y que es regulado por las normativas vigentes establecidas. Al respecto, el liderazgo en el centro es necesario para lograr conectar las expectativas de los docentes con las del proceso de formación y el plan del centro, para que con el apoyo de todos los miembros de la comunidad educativa y una idea clara de lo que se desee hacer se transforman los mismos. Destacando adicionalmente que:

- Cambió la organización del centro antes y después de la formación

- Se creó un aula de plástica nueva

- Se logró contar con personas interesadas en el proyecto que se realiza

- Hay un equipo directivo con unas directrices de lo que se debe llevar a cabo

- Las experiencias del centro parten de la demanda del centro de formación

- Aplican alternativas de solución a situaciones disfuncionales

- Promueve la institucionalización de las buenas prácticas

- La realidad ha ido imponiendo la existencia de equipos directivos y equipos impulsores del cambio y de la transformación

A partir de las ideas anteriores, se considera que las instituciones objeto de investigación realizan importantes esfuerzos en la formación del docente a través de las artes; los cuales se van a consolidar a medida que todos se involucren, participen y apliquen los resultados en la praxis educativa.

\subsection{Grupo Focal}

El grupo focal contó con la participación de las tres personas del equipo directivo: directora, jefa de estudios y secretaria, como también la especialista de visual y plástica y la investigadora, coincidiendo en que se había valorado de forma positiva la formación. Se realizó, asimismo, considerando las opiniones que el equipo impulsor brindaba sobre los itinerarios por lo que es importante destacar que les permitió tomar conciencia de aquello que deben cambiar y que en su momento formaba parte de la normalidad del centro.

El grupo se reunió 4 veces con cada uno de los dos centros educativos durante el curso escolar en la misma escuela, con un total de 8 reuniones durante los dos cursos que duró el proceso de recogida de datos. Las reuniones se distribuyeron de la siguiente modo: 1 principio de curso, 2 durante el proceso de transferencia al centro, y 1 de conclusiones a final de curso además, si hicieron distintas reuniones semanales antes de la formación presencial mientras duró la formación de los Itinerarios Artísticos.

Se determinó que los docentes requerían formación, pero que ésta fuera especializada y que les permitiera desarrollarse. La educación artística, por sus particularidades, les permite aplicar la innovación y la creatividad a 
todos los ámbitos educativos; lo cual justificó esta investigación en su orientación y fundamentación, sensibilizándolos en la necesidad de un cambio del claustro para generar una transformación personal y que sería llevada al alumnado en su beneficio. Este proceso de reflexión les permitió evidenciar sus debilidades y aspectos por mejorar en la praxis profesional con el apoyo del equipo impulsor del centro y los itinerarios

Figura 6

Grupo focal

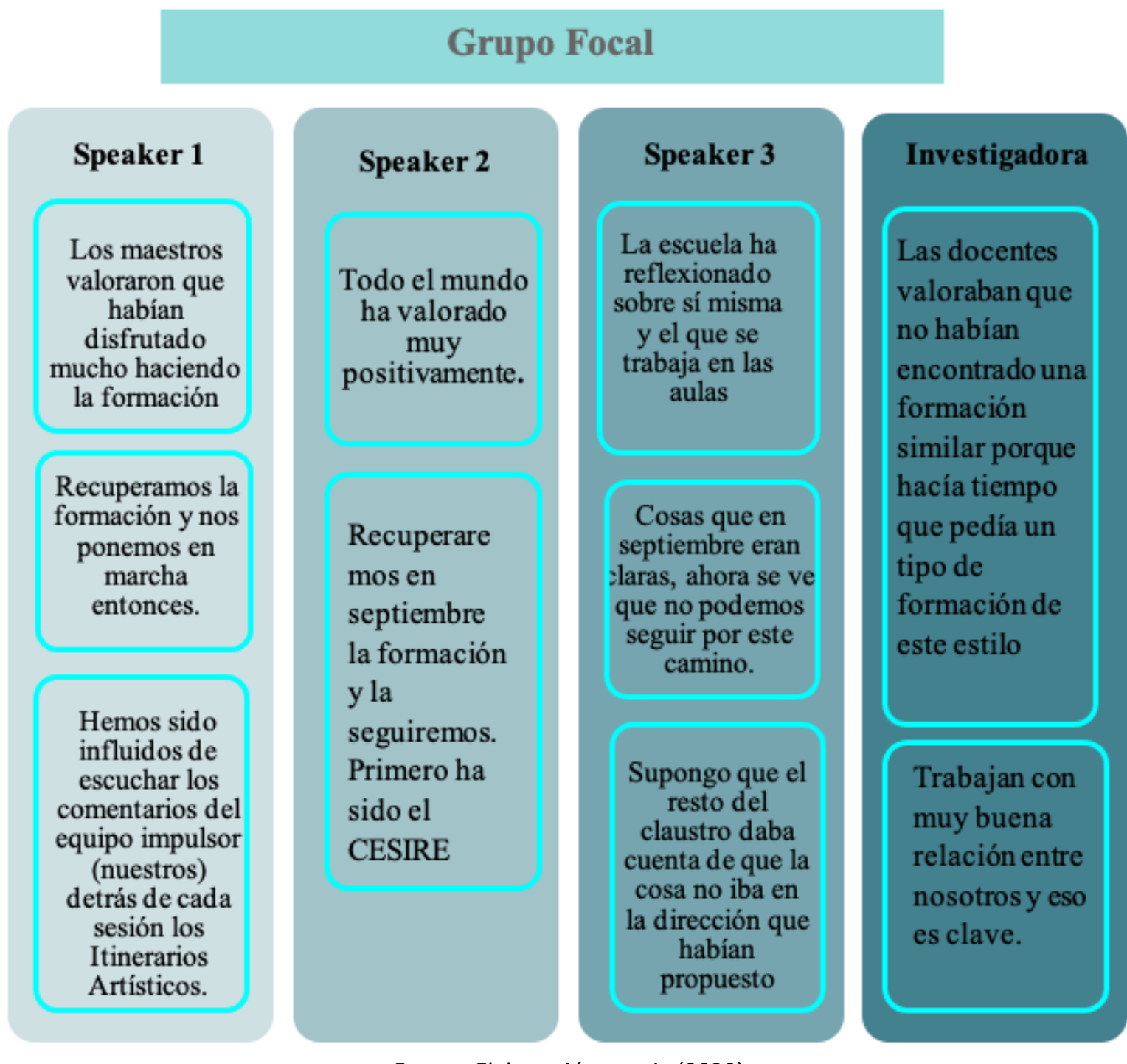

Fuente. Elaboración propia (2020)

\subsection{Guia de observación}

Los resultados que se presentan en la hoja de observación corresponden a los itinerarios de cada uno de los centros y los resultados presentados en las rúbricas de cada uno de ellos con relación al proceso de formación docente. Comparando ambos centros, se puede apreciar que la Escuela Ferrer i Guàrdia Granollers muestra mejores resultados en los itinerarios artísticos y proceso de formación continua de docentes.

Cada itinerario estuvo configurado o construido a partir de la selección de cápsulas o unidades de formación, siendo en sí mismo una propuesta original e innovadora que se dispone y adapta a las necesidades e intereses 
de los participantes desde una mirada competencial del aprendizaje. El itinerario se plantea como una propuesta que se diseña y administra a través del CESIRE, para hacer accesible el desarrollo de propuestas metodológicas, que facilitan la investigación y la indagación a través de la composición de los grupos de arte. En este ámbito, las experiencias académicas de los centros fueron positivas en los procesos de formación docente a través del uso de la educación artística, generando cambios en los mismos y transformación en la praxis académica en la educación primaria.

En este proceso formativo los participantes son los actores principales de una propuesta planteada como un escenario abierto, que promueva el desarrollo del proceso creativo, en tanto docente individual o como parte de la comunidad educativa. Para esto se posibilita realizar dos tipos de inscripción: individual o como claustro. Ambas deben funcionar a través de la adaptación a las necesidades, los objetivos del centro y a su identidad Generalitat de Catalunya (2020). En esta investigación, se optó por la formación como claustro. Asimismo, durante el desarrollo de los procesos de formación, los diferentes ejercicios se representan de manera dinámica mediante los itinerarios artísticos a través de sus cápsulas. Éstos se convierten en espacios de aprendizaje caracterizados por ser propuestas originales, con temáticas y actividades innovadoras que se aproximan al caos para dar rienda suelta a la manifestación de los procesos de creatividad. Todo esto transforma al docente sensibilizándolo en cuanto al arte y aplicando todos estos principios al currículo de este nivel.

\section{Conclusiones}

En el primer objetivo "Mostrar los resultados de experiencias académicas fundamentadas en el uso de la educación artística en los procesos de formación dirigidos a docentes" se evidenció, en las apreciaciones del personal de los centros y de la investigadora, que las experiencias académicas fueron positivas para el docente y la institución, permitiendo llevar a cabo ajustes metodológicos, teóricos y prácticos que en el futuro beneficiarán a los niños y niñas de los centros no sólo en este ámbito, sino también en otros.

Todos estos elementos destacan la relevancia de la educación artística para los procesos de formación docente en educación primaria, a través de los Itinerarios Artísticos Autoplanificados mediante los cuales es posible desarrollar actividades novedosas e innovadoras con el profesorado. La educación artística se convierte así en un instrumento mediante el cual se logra este objetivo, permitiendo valorar en cuanto a su importancia en el proceso de enseñanza y aprendizaje en los centros educativos. De esta manera, los objetivos e identidad de ambos centros convergen en cuanto a la aplicación internalizando en los valores y objetivos de cada centro.

En cuanto al segundo objetivo: Indagar los referentes teóricos que sustentan los procesos académicos relacionados con la dinamización de los procesos de educación continua a través de la educación artística, éstos se evidencian a través de la revisión de fuentes teóricas consultadas. Esta revisión evidencia que la educación artística y la formación docente cuenta con una amplia gama de autores relevantes presentados en el desarrollo de esta investigación, y que permitieron sustentar el proceso de análisis e interpretación de la información.

Finalmente, en el tercer objetivo: Contrastar la influencia que tiene el desarrollo de procesos de educación continua, centrados en la educación artística para docentes, en el desarrollo de los procesos formativos de sus estudiantes de educación infantil y primaria en el contexto de transformación de centro a través de las artes, se demostró que existe una influencia directa entre los procesos de educación o formación continua de los docentes y los estudiantes, constatando que al cambiar la forma de visualizar la praxis educativa mejora la atención al alumnado y la calidad de la educación. Desde el punto de vista teórico esto se demostró en las investigaciones de García (2016) dando cuenta de que su utilización para la dinamización de los procesos de formación continua permite conseguir los resultados esperados, demostrando así que la aplicación de su propuesta mejora el proceso de formación continua del profesorado en las dimensiones personal, pedagógica y social-comunitaria. Ocampo-Gómez \& Souto (2012) han reafirmado esta postura cuestionándose sobre la forma en que la 
experiencia profesional y personal de los maestros influyen en la forma de abordar a los hijos escolarizados migrantes.

También es importante destacar que esta formación les permitió tomar conciencia de aquello que debían cambiar y que en su momento formaba parte de la normalidad del centro. Del mismo modo, se concluye que esta investigación fue una oportunidad, un motor y una palanca de cambio para potenciar los procesos de enseñanza y aprendizaje de docentes y estudiantes en relación con los elementos de expresión artística y la transformación de la cultura del centro.

En conclusión, partiendo de que el aprendizaje es dado a partir de los diálogos en contextos sociales, culturales y materiales, el desarrollo de los procesos formativos y de aprehensión de conocimiento durante la formación continua se desarrolló en interacción y colaboración dentro de una comunidad de aprendizaje que compartía prácticas comunes centradas en un determinado aspecto o tema y orientadas en el mismo objetivo común de transformar el propio centro a través de las artes. En nuestras vidas cotidianas cómo docentes estamos y somos miembros de múltiples contextos simultáneamente por lo que nuestras identidades están compuestas por distintas capas interrelacionadas de forma compleja. (Forné, 2012). Tal y como afirmó New London Group (2000) el rol de la pedagogía es desarrollar una epistemología del pluralismo sin que las personas se vean en la encrucijada de abandonar sus subjetividades. Es decir, de acuerdo con estos autores, docentes y alumnos, necesitan un lenguaje específico para describir las formas de significación, y la formación continua en la que participaron se la facilitó, así como también los contextos facilitadores para que ello sucediera.

\section{Referencias bibliográficas}

Acaso, M. (2009). La educación artística no son manualidades. Nuevas prácticas en la enseñanza de las artes y la cultura visual. Madrid: Catarata.

Aguirre, I. (2005). Teorías y Prácticas en Educación Artística. Ideas para una revisión pragmatista de la experiencia estética. Barcelona: Octaedro

Aznárez, J. P. (2018). La necesidad de educar en artes visuales. Arte y movimiento, 18. 17-41. (18). Recuperado de https://revistaselectronicas.ujaen.es/index.php/artymov/article/view/3914

Ballester, L.L., Nadal, C., \& Amer, J. (2014). Métodos y técnicas de investigación educativa. Madrid: UIB.

Bamford, A. (2009). El factor iwuau! El papel de las artes en la educación. Madrid: Octaedro.

Bogdan, R.C., \& Biklen, S.K. (1982). Qualitative research for education: An introduction to theory and methods. Allyn \& Bacon.

Departament d'Educació, (2019-2020). Criteris, instruccions i orientacions.Formació permanent del professorat. https://serveiseducatius.xtec.cat/tarragones/wp-content/uploads/usu549/bpattachments/14045/PFZ-Criteris-i-Instruccions-2019-2020.pdf

Deleuze, G. y Guattari, F. (2002). Mil mesetas. Capitalismo y esquizofrenia. Madrid: Pre-Textos.

Eisner, E.W. (2002). The Arts and the Creation of Mind. Yale University Press \& New Haven and London. Harrisonburg: R.R.Donnelley \& Sons

Eisner, E.W. (2007). Cognición y Currículum: Una visión nueva. Madrid:Amorrortu

Forné, E. (2012). Ítaca, un periplo de indagación identitaria a través de los Nuevos Alfabetismos. El papel del Alfabetismo Medial y visual en la construcción de identidades. [Trabajo Fin de Máster]. Máster de Artes Visuales y Educación: un enfoque construccionista. Facultad de Bellas Artes. Universidad de Barcelona. 
Glaser, B. \& Strauss, A. (2012). The Discovery of Grounded Theory: Strategies for Qualitative Research. Aldine Transactions. A Division of Transaction Publishers.

García, E. (2016). Programa de capacitación docente crítico, reflexivo y democrático para mejorar la formación continua de las profesoras, en la Institución Educativa Estatal №1600 de la Provincia de Pacasmayo2015. [Tesis doctoral, Universidad Nacional de Educación Enrique Guzmán y Valle]. Recuperado de http://repositorio.une.edu.pe/handle/UNE/924

González, J.M., Arquero, J.L. \& Hassal, T. (2014). Consolidación de la formación por competencias en la universidad española: Estudio de un caso. Educación XX1, 17(2), 145-168. https://www.redalyc.org/pdf/706/70630580008.pdf

Husserl, E. (1966). The Phenomenology of Internal Time-Consciousness. Indiana University Press.

Kennedy (2005). Models of Continuing Professional Development. Journal of In-service Education ,31(2), 235250. Recuperado de https://www.researchgate.net/publication/232902672_Models_of_Continuing_Professional_Developmen t_A_framework_for_analysis

Latorre, A.,et.al. (2005) Investigación Educativa. Metodologías de investigación educativa. Mexico: Experiencia, S.L.

Llonch N., et.al. (2017). Una experiencia de formación del profesorado basada en las inteligencias múltiples y la Educación Artística. Educatio Siglo XXI, 35, 317-340. Recuperado de https://www.researchgate.net/publication/321249296_Una_experiencia_de_formacion_del_profesorado _basada_en_las_inteligencias_multiples_y_la_Educacion_Artistica

Monereo, (2010). La formación del profesorado: una pauta para el análisis de intervención a través de incidentes críticos. Revista Iberoamericana de educación, 52, 149-178. Recuperado de https://rieoei.org/historico/documentos/rie52a08.pdf

Ocampo-Gómez, C.I. \& Souto, B. (2012). Formación, experiencia docente y actitudes de los profesores de infantil y primaria ante la educación escolar de hijos de personas inmigrantes en España. Revista de investigación educativa, RIE. 30(1), 111-130. Recuperado de https://dialnet.unirioja.es/servlet/articulo?codigo=3837223\&orden=0\&info=link

Rivera, E.F. (2013). Estructuras de sentido de la educación artística en la formación de maestros. Historia de la Educación Colombiana, (16), 18. Recuperado de https://revistas.udenar.edu.co/index.php/rhec/article/view/1748

Rojas, B. (2014). Investigación Cualitativa. Fundamentos y praxis. Fedupel

Rodríguez, P. \& Bonilla E. (2005). Más allá del dilema de los Métodos. Norma.

Sandín, M.P. (2003). Investigación cualitativa en educación. Fundamentos y tradiciones. Madrid: McGraw-Hill.

Vázquez, R. \& Angulo, F. (2003). Introducción a los Estudios de Casos. Los primeros contactos con la investigación etnográfica. Madrid: Aljibe.

Vaillant, D. \& Marcelo, C. (2015). El ABC y D de la formación docente. Educación Hoy Estudios,134. Madrid:Narcea.

Vilaboa, D.R. (2005). Educación plástica y artística en educación infantil: una metodología para el desarrollo de la creatividad. Ideas propias: Editorial SL.

Esta obra está bajo una Licencia Creative Commons Attribución-NoCommercial 4.0 International

(cc) BY-NC 